OPEN

SUBJECT AREAS:

PLANT SCIENCES

BIOPHYSICS

Received

3 December 2013

Accepted

5 June 2014

Published

25 June 2014

Correspondence and requests for materials should be addressed to

L.H. (hlan@cau.edu. cn, biomed_hl@263.

net)

\section{Spatio-temporal mapping of variation potentials in leaves of Helianthus annuus L. seedlings in situ using multi-electrode} array

\author{
Dong-Jie Zhao' , Zhong-Yi Wang' ' Lan Huang' ', Yong-Peng Jia ${ }^{2} \&$ John Q. Leng ${ }^{3}$
}

${ }^{1}$ College of Information and Electrical Engineering, China Agricultural University, Beijing 100083, China, ${ }^{2}$ Bio-gene Technology Ltd., Beijing 100124, China, ${ }^{3}$ Department of Cellular and Molecular Physiology, Yale University School of Medicine, New Haven, CT 06520, USA.

Damaging thermal stimuli trigger long-lasting variation potentials (VPs) in higher plants. Owing to limitations in conventional plant electrophysiological recording techniques, recorded signals are composed of signals originating from all of the cells that are connected to an electrode. This limitation does not enable detailed spatio-temporal distributions of transmission and electrical activities in plants to be visualised. Multi-electrode array (MEA) enables the recording and imaging of dynamic spatio-temporal electrical activities in higher plants. Here, we used an $8 \times 8 \mathrm{MEA}$ with a polar distance of $450 \mu \mathrm{m}$ to measure electrical activities from numerous cells simultaneously. The mapping of the data that were recorded from the MEA revealed the transfer mode of the thermally induced VPs in the leaves of Helianthus annuus L. seedlings in situ. These results suggest that MEA can enable recordings with high spatio-temporal resolution that facilitate the determination of the bioelectrical response mode of higher plants under stress.

\footnotetext{
- lectrical signals have been found in many plant species, including lower plants and both sensitive and non-sensitive higher plants. These signals are involved in many plant biological processes such as respiration $^{1}$, water uptake ${ }^{2}$, leaf movement ${ }^{3}$, and biotic stress responses ${ }^{4}$. The two main electrical signals that are found in plants are action potentials (APs) and variation potentials (VPs). While APs are rapid signals that are induced by non-damaging stimuli ${ }^{5}$, VPs are slower signals that are induced by damaging stimuli $^{6,7}$ (e.g., burning and cutting). Indeed, VPs are sometimes called slow-wave potentials because their transmission speed is typically slower than that of $\mathrm{APs}^{8}$. Additionally, VPs decrease in magnitude as they spread away from stimulated sites ${ }^{9}$. Both types of signals carry information to other cells, tissues, and organs, where they trigger appropriate responses. However, the modulation of VP amplitude, which is accompanied by interdependent ion fluxes ${ }^{10}$, increased ethylene emissions ${ }^{11}$, and induced proteinase-inhibitor gene expression $^{12}$, may enable the VPs to carry more information than the APs. In fact, VPs that are induced by different stimuli have distinguishing signal characteristics and propagation patterns ${ }^{13}$. However, compared with APs, the patterns and pathways of VP transmission are less well known. Because of limitations in recording wound-induced VPs by surface electrodes, distinguishing changes in electrical potentials from multiple cells or tissues is impossible in plants, as is the mapping of VP propagation.

Multi-electrode arrays (MEAs) can overcome these limitations and enable the monitoring of bioelectrical activities at multiple sites with high spatio-temporal resolution ${ }^{14}$. MEAs have been used in vitro for more than 40 years to measure the electrical activities of heart cells from cultured embryonic chicks ${ }^{15}$. A MEA device consists of an array of microelectrodes (typically $10-50 \mu \mathrm{m}$ in diameter) that are connected to an external circuitry that enables the recording of electrical activities from excitable cells (usually neurons or cardiomyocytes) and tissues. In recent years, significant progress has been made in this technique, and highly reliable MEAs have been used in the extracellular and intracellular recording of animal cells ${ }^{16-19}$ and maize root apices ${ }^{20}$. Here, we employed an $8 \times$ 8 MEA to measure VPs in H. annuus leaves in vivo and in situ and investigated the propagation of VPs in a region of interest (ROI).
} 


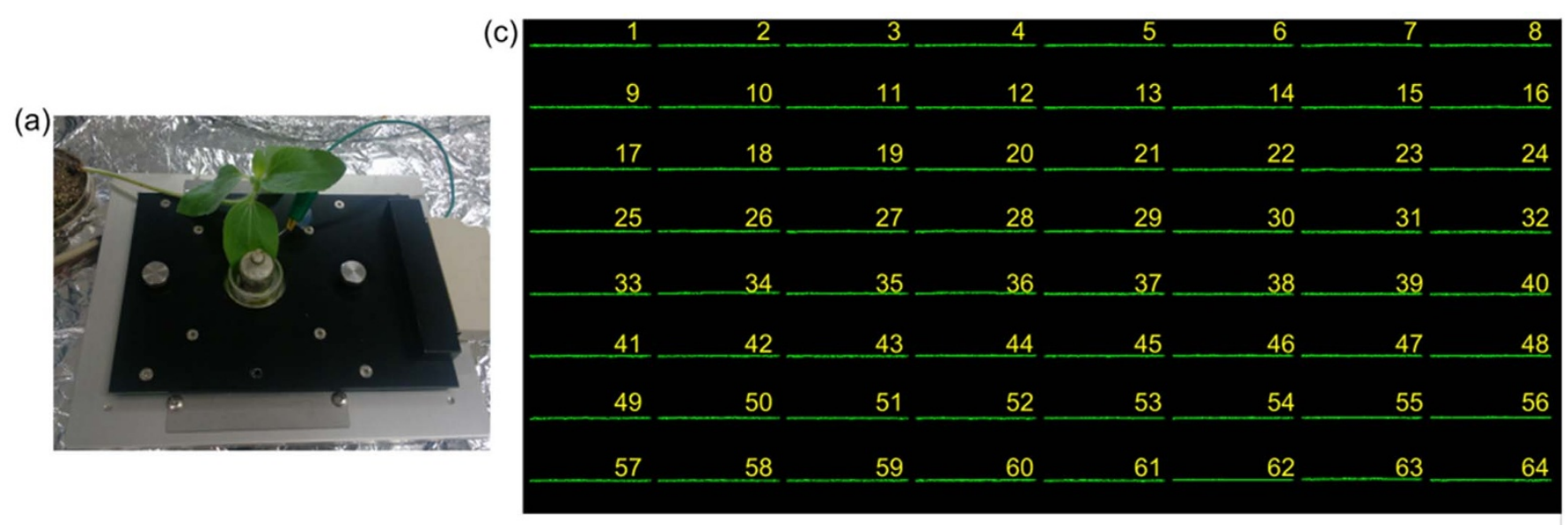

(b)

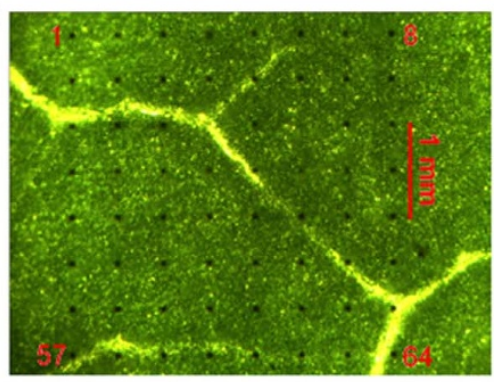

(d)

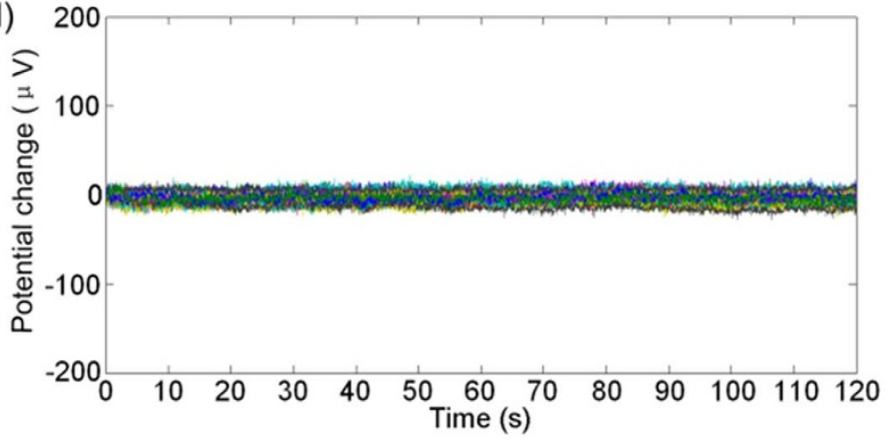

Figure 1 Coupling of a $\mathrm{H}$. annuus leaf and electrodes of the MED64 array. (a) The leaf was placed on the electrode panel, and a U-shaped platinum plate with a grid of nylon threads was placed on the top of the leaf. A 25-g weight that was sealed with a plastic membrane was then placed on the platinum plate. A $10 \mathrm{mM} \mathrm{KCl}$ solution was placed in the electrode chamber. (b) A microscopic view of the planar electrodes and leaf after recording, showing the close connections between them; the black dots are the electrodes. (c) Baseline activities of 64 electrodes after coupling. (d) Overlapped baseline signals of the 64 channels that are shown in (c), indicating that the noise level was less than $\pm 17 \mu \mathrm{V}$.

\section{Results}

Noise level of the MED64 array. Figure 1a shows the coupling of electrodes in the array and a $H$. annuus leaf. Figure $1 \mathrm{~b}$ shows a closeup view of the connection between the planar electrodes and the leaf. In the present study, we found that the ion concentration of a solution in the probe chamber had an effect on the noise level of the recording results. The recording results with different $\mathrm{KCl}$ concentrations $(1 \mathrm{mM}, 2 \mathrm{mM}, 5 \mathrm{mM}$, and $10 \mathrm{mM})$ and the standard buffer solution $\left(2 \mathrm{mM} \mathrm{KCl}, 1 \mathrm{mM} \mathrm{CaCl}, 1 \mathrm{mM} \mathrm{MgCl}_{2}, 50 \mathrm{mM}\right.$ mannitol, and $2.5 \mathrm{mM} \mathrm{MES} / \mathrm{NaOH}$ buffer, $\mathrm{pH}$ 5.7) demonstrated a noise level of approximately $\pm 50 \mu \mathrm{V}$ in $1 \mathrm{mM} \mathrm{KCl}$, approximately $\pm 17 \mu \mathrm{V}$ in $10 \mathrm{mM} \mathrm{KCl}$, and approximately $\pm 18 \mu \mathrm{V}$ using a standard buffer solution (see Supplementary Fig. S1). Thus, $10 \mathrm{mM} \mathrm{KCl}$ and the standard buffer solution were used in the experiments, respectively. Figure 1c and Figure $1 \mathrm{~d}$ show the baseline and noise level of the MED64 array. The noise level was less than $\pm 17 \mu \mathrm{V}$ at a band-pass range of $0.1-1 \mathrm{KHz}$ and a sampling frequency of $20 \mathrm{KHz}$ at $10 \mathrm{mM} \mathrm{K}^{+}$.

Recording of variation potentials using MEA and analyses of signal transmissions. We recorded heat-induced electrical responses from 45 different plants in distinct experiments (38 plants for recording in $10 \mathrm{mM} \mathrm{KCl}$ and 7 plants for recording in standard buffer solution) and observed similar transmission patterns for all of the experiments. A typical example of a response to thermal stimulation is illustrated in Fig. 2. Figure 2a indicates the relative position between the planar microelectrodes and the $H$. annuus leaf. We stimulated the right leaf and recorded the left leaf. Figure $2 \mathrm{~b}$ shows the field potential that was induced by thermal stimulation, and nearly all 64 of the electrodes successfully recorded VPs. In Fig. 2c, the signals from Fig. $2 \mathrm{~b}$ are overlapped, showing that the VPs occurred with a mean latency of $55 \pm 5 \mathrm{~s}$ after stimulation onset, which lasted $30 \pm 2$ s. Similar response times have been reported in other studies ${ }^{6}$. The signal amplitudes were in the range of $50-400 \mu \mathrm{V} \mathrm{Vp-p.} \mathrm{Clear} \mathrm{delays} \mathrm{can} \mathrm{be} \mathrm{seen} \mathrm{among} \mathrm{the}$ different VP traces, indicating that VP transmission patterns differed depending on the ROI. To determine the propagation directions and transmission routes of the VPs within the recording region, we spatially mapped each VP according to its placement within the MEA of the electrode that recorded it. The direction of propagation is shown by the colour coding of the VPs according to their onset time in Fig. 2d (blue, early onset; yellow/green, mid-range onset; red, late onset). The colour coding revealed that the VPs first occurred on the right side (electrodes $8,16,24,32,40,48$, and 56) of the MEA and gradually progressed to the upper left side (electrode 1). The time delay between the first and last VP onset (electrodes 8 and 1, respectively) was $6 \mathrm{~s}$. The propagation velocities of the VPs in any direction can be easily deduced from the time delay between the detection of the signal at the two selected electrodes (shown by arrows in Fig. 2e). The propagation speeds ranged from $0.7 \mathrm{~mm} / \mathrm{s}$ to $2.5 \mathrm{~mm} / \mathrm{s}$ and occurred in different directions. The VP that was transmitted along the main propagation route (red arrow, Fig. 2e) had a slower propagation velocity than those of the VPs that were transmitted along the branching routes (blue arrows, Fig. 2e). Considering the relative positions of the electrodes on the leaf (Fig. 2a), the VPs were clearly induced in the right leaf, transmitted along the petiole to the left leaf, and subsequently travelled across the surface of the left leaf. Figure $2 \mathrm{f}$ uses time-lapse pseudocolour images to illustrate the amplitude changes that occurred as the VPs propagated across the leaf. The potential change initially 
(a)
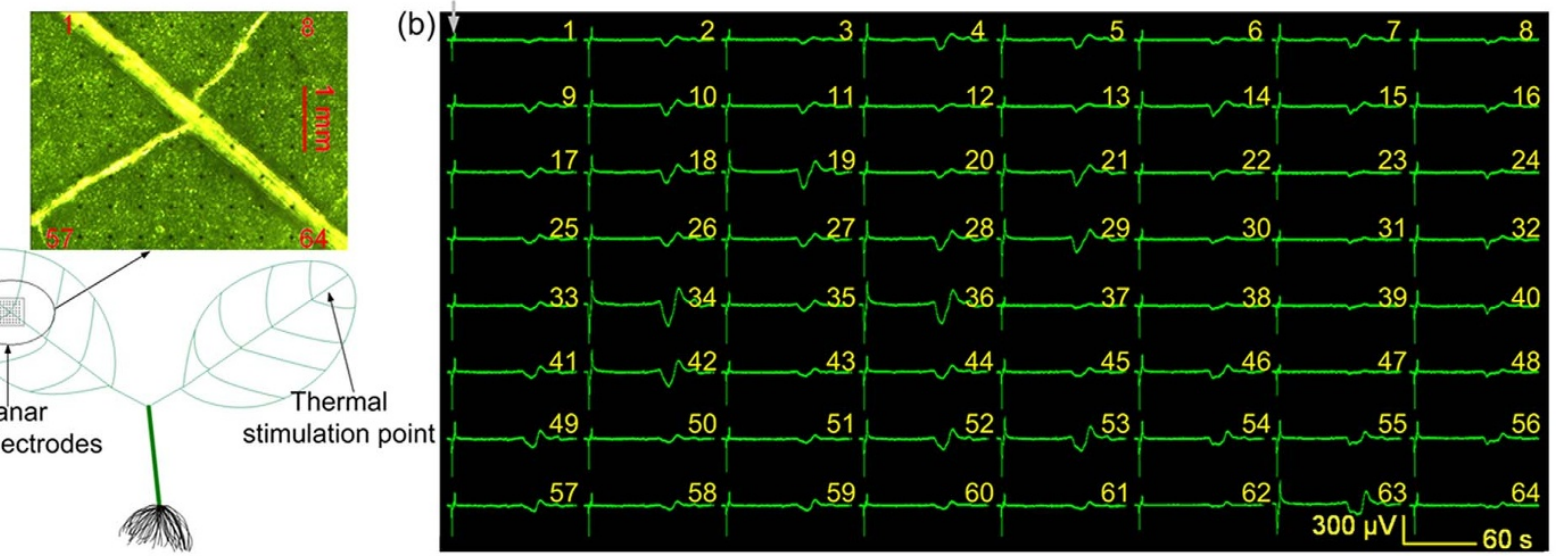

(c)
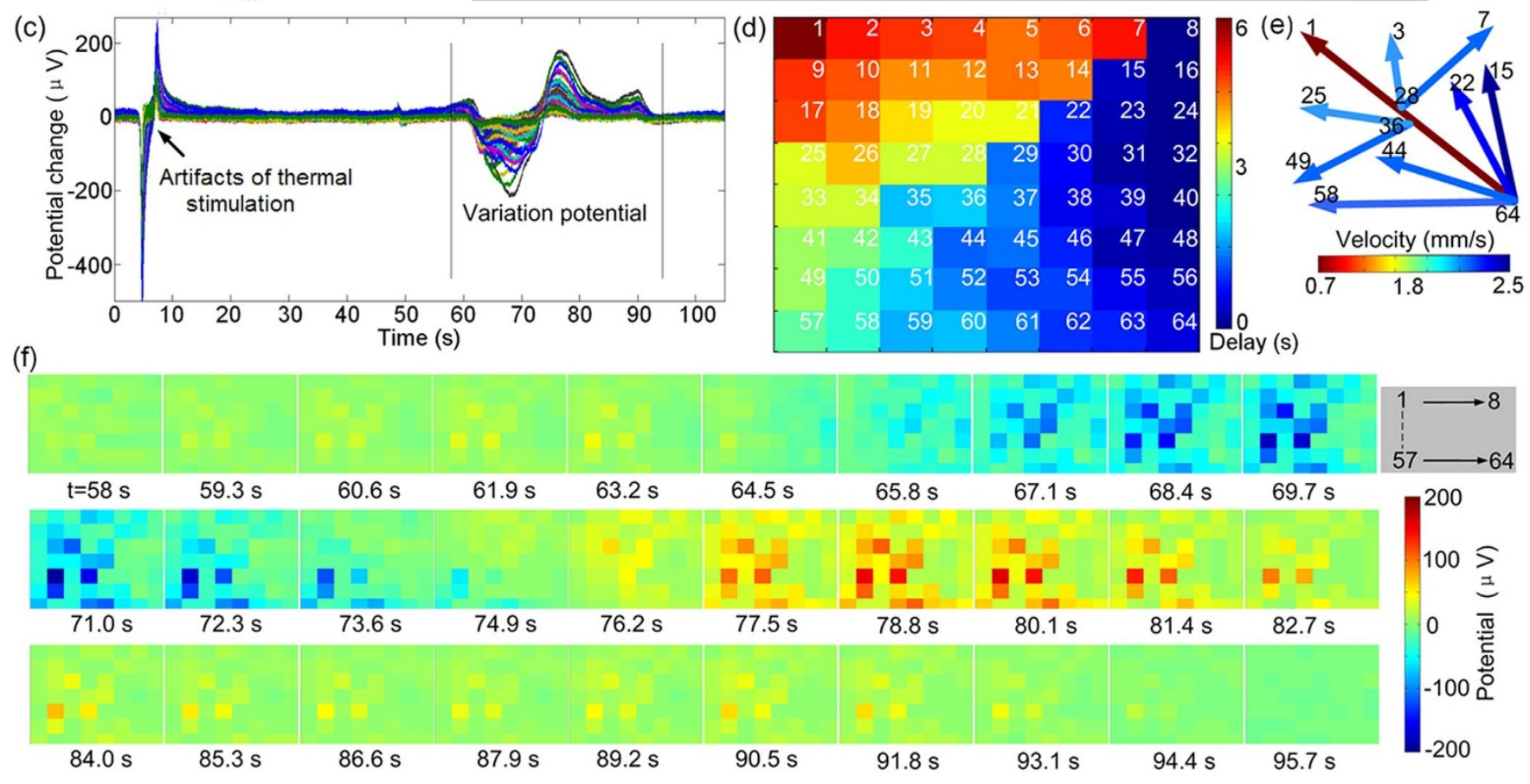

Figure 2 Spatio-temporal propagation of waves in a leaf neighbouring the heat-stimulated leaf. (a) Relative position of planar microelectrodes (left leaf) and location of stimulation (right leaf). (b) After thermal stimulation, nearly all 64 of the electrodes recorded VPs. The white arrow indicates an artifact that was observed during the thermal stimulation. (c) Overlapping signals from (b). The VP onset was delayed approximately $50 \mathrm{~s}$ after stimulation, and the VP duration was approximately $30 \mathrm{~s}$. (d) The VP propagation was visualised through the colour-coding of the VP onset at each electrode. The VPs reached the electrodes that are depicted in blue first and then propagated to the yellow regions and finally to the red regions.

(e) Average VP propagation velocities in the different directions. The velocity equals the distance between two numbered planar electrodes on the arrow divided by the time delay between signal detection at the two electrodes. (f) Maps of the voltage distributions for the propagating waves at individual snapshots in time. Changes in voltage partially match the propagation direction (right to left) that is shown in (d).

occurred in the right recording side (blue squares, $t=64.5 \mathrm{~s}$ ) and then spread to the left, which is a pattern that partially matches the transmission direction that is seen in Fig. 2 d.

The results from the experiment shown in Fig. 3 cross-validate those from the experiment shown in Fig. 2. Because thermal stimulation damages the plant, we used different $H$. annuus samples in this experiment. Compared with Fig. 2a, the relative positions for recording and stimulation were opposite. Again, nearly all 64 of the electrodes recorded VPs that were induced in the left leaf by the thermal stimulation of the right leaf (Fig. 3b), and the delay time was approximately $40 \mathrm{~s}$ (Fig. 3c), which is similar to the results obtained in the first experiment. Although the 20-s duration was shorter than that in the first experiment, the times were comparable. Additionally, variations in onset delay can be seen across the electrodes. For example, the delay time was $5 \mathrm{~s}$ between electrodes 25 and 40 (Fig. 3d). The propagation speeds were also similar to those that are illustrated in Fig. 2, measuring from $0.8 \mathrm{~mm} / \mathrm{s}$ along the main route (red arrow in
Fig. 3e) to $2 \mathrm{~mm} / \mathrm{s}$ along a branching route (blue arrow in Fig. 3e). Figure $3 \mathrm{~d}$ and Figure $3 \mathrm{f}$ show that the VPs first reached electrodes on the left side of the grid and then travelled to the right side, which was again consistent with the experimental setup, indicating that the VPs spread with characteristic patterns across the leaf surfaces and from one leaf to another. Additionally, we recorded the leaf VPs in exactly the same way for the MED probe with the standard buffer solution. The results are consistent with those that are shown in Fig. 2 and Fig. 3 (see Supplementary Fig. S2).

Furthermore, we used conventional inhibitors of $\mathrm{H}^{+}$-ATPase and ion channels, including sodium orthovanadate, $\mathrm{La}^{3+}$, and tetraethylammonium ion $\left(\mathrm{TEA}^{+}\right)$, to examine this MEA recording method. The VPs of the leaves that were treated with inhibitors as recorded by MED64 are shown in Supplementary Fig. S3. The profiles in Supplementary Figs. S3a, b, and c show the VP recorded approximately $70 \mathrm{~s}$ after heat stimulation. The maximum delay times of the leaves that were treated with sodium orthovanadate increased to 7.3 
(a)

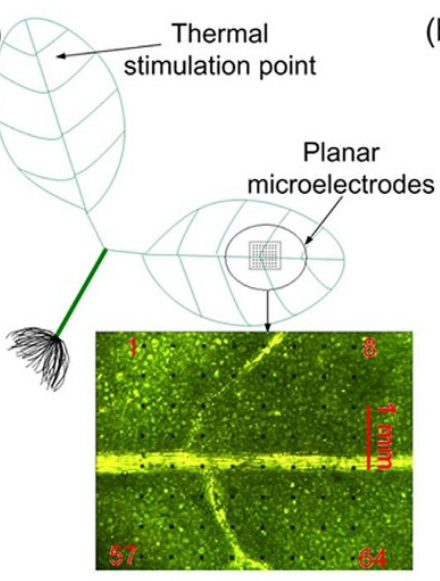

(c)

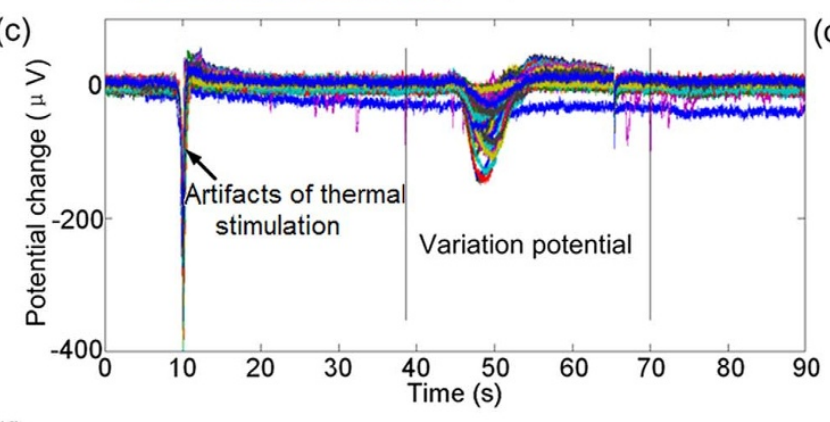

(b)

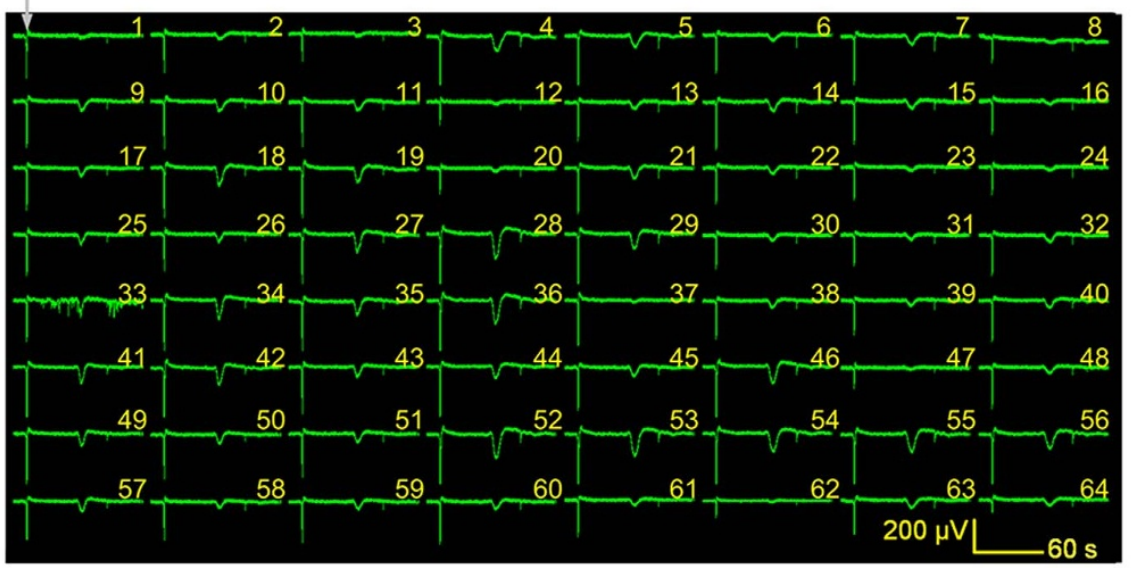

.

(1)

(d)

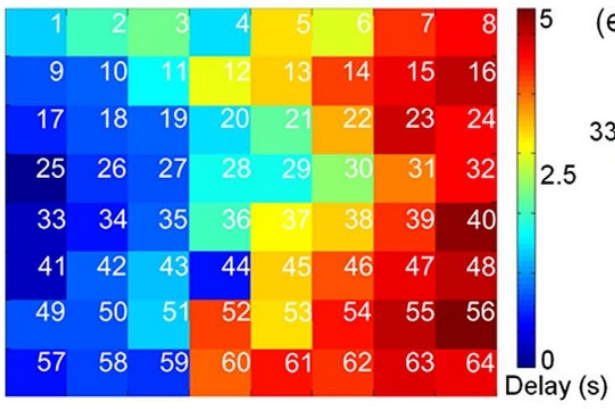

(e) 3

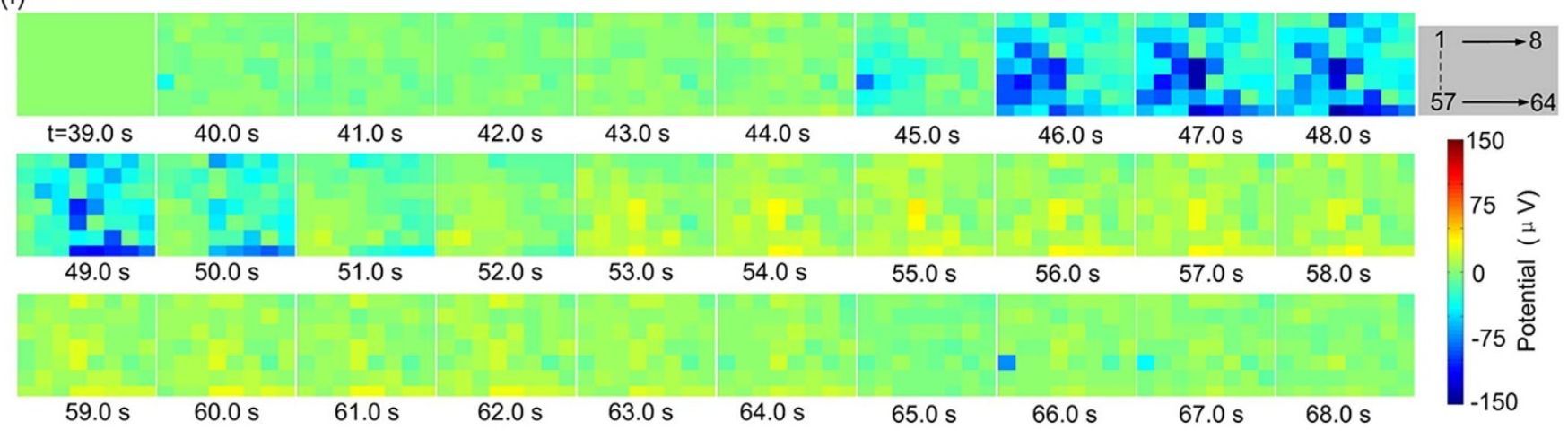

Figure 3 Spatio-temporal propagation of waves in a leaf neighbouring the heat-stimulated leaf; cross validation of results using a different plant. (a) Relative positions of the planar microelectrodes (right leaf) and the stimulation location (left leaf). (b) After thermal stimulation, nearly all 64 of the electrodes recorded VPs. The white arrow indicates the artifact that was observed during the thermal stimulation. (c) Overlapped signals from (b).

(d) The VP propagation was visualised through the colour coding of the VP onset at each electrode. The VPs reached the electrodes that are depicted in blue first, then propagated to the yellow regions, and finally moved to the red regions. (e) Average VP propagation velocities in different directions. The velocities were calculated as described in the caption of Fig. 2e. The propagation velocity was slower along the main route than along the branching routes. (f) Maps of the voltage distributions for the propagating waves at individual time points. Changes in voltage match the propagation direction (left to right) that is shown in (d).

$\pm 1.5 \mathrm{~s}, 6.3 \pm 1.4 \mathrm{~s}\left(\mathrm{La}^{3+}\right)$, and $7.2 \pm 1.3 \mathrm{~s}\left(\mathrm{TEA}^{+}\right)$, compared with the control delay time of $5.5 \pm 1.7 \mathrm{~s}$. Although there is a slight difference between the maximum delay time in Supplementary Fig. S3d and that in Fig. 2, the mean velocities ranged from $0.6 \mathrm{~mm} / \mathrm{s}$ in the main route to $2 \mathrm{~mm} / \mathrm{s}$ in the branch route, which are smaller than those shown in Fig. 2 and Fig. 3. The maps of the voltage distributions for the propagating waves at individual time points are displayed in Supplementary Fig. S3f, which shows that the amplitudes of the VPs after inhibition are much smaller than those of the controls. All of the blockers were shown to significantly reduce the amplitudes of the VPs compared with the control $(p<0.05)$ (Fig. 4a) and increase the maximum delay times among the signals, although these increases were not significant (Fig. 4b). As shown in Fig. 4, the $\mathrm{H}^{+}$-ATPase and ion channel blockers altered the amplitudes of the VPs from control values of $249 \pm 51 \mu \mathrm{V}$ to $71 \pm 13 \mu \mathrm{V}$ (1 mM sodium orthovana- date), $67 \pm 15 \mu \mathrm{V}\left(1 \mathrm{mM} \mathrm{La}^{3+}\right)$, and $56 \pm 15 \mu \mathrm{V}\left(10 \mathrm{mM} \mathrm{TEA}^{+}\right)$. In addition, the recorded leaf petioles were killed by the $10 \%$ paraformaldehyde solution, no VPs were recorded for those leaves $(n=8)$ (see Supplementary Fig. S4).

\section{Discussion}

Using a MEA, we recorded the VPs in the leaves of H. annuus in vivo at multiple sites simultaneously. In contrast to surface electrodes, MEA has high spatio-temporal resolution that can detect changes in potentials in a $3.55 \mathrm{~mm} \times 3.55 \mathrm{~mm}$ area using 64 planar microelectrodes.

In our experiments, the VP amplitudes were in the range of 50$400 \mu \mathrm{V}$. Compared with traditional extracellular recording using surface electrodes, in which VP amplitudes usually range from several millivolts to tens of millivolts ${ }^{6,21}$, the amplitudes that were 
(a)

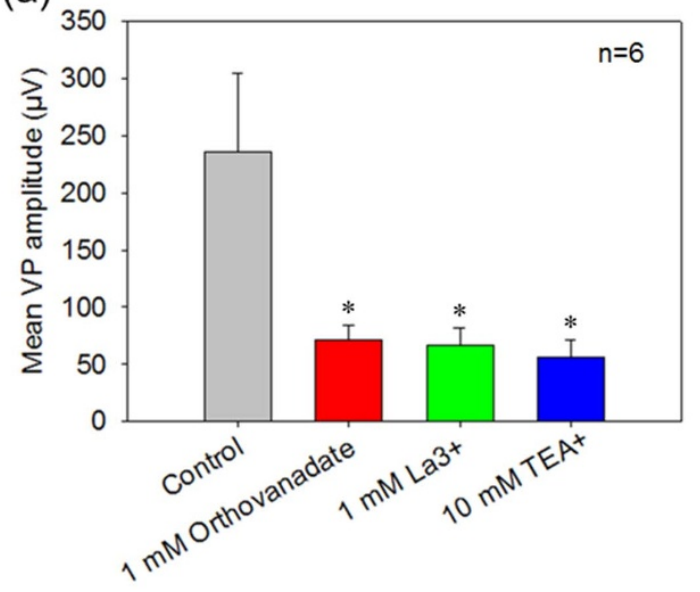

(b)

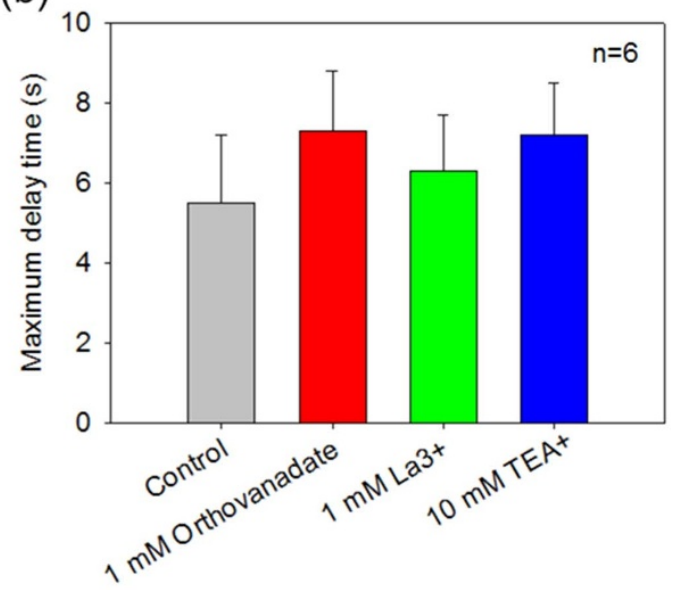

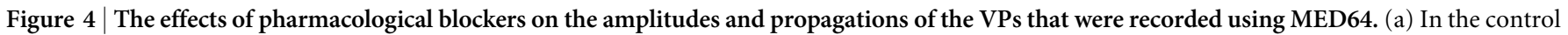

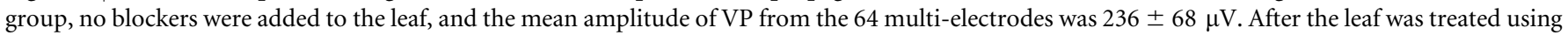

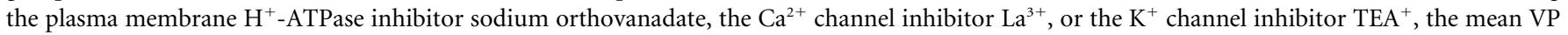
amplitude was reduced to $71 \pm 13 \mu \mathrm{V}, 67 \pm 15 \mu \mathrm{V}$, and $56 \pm 15 \mu \mathrm{V}$, respectively. Data are presented as the mean \pm S.E.M. unless stated otherwise

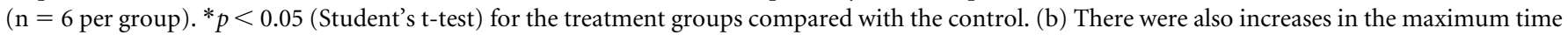

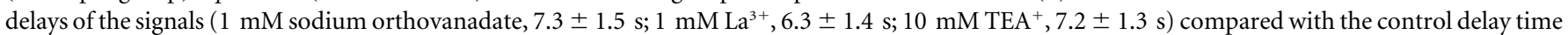
$(5.5 \pm 1.7 \mathrm{~s})$. Data are presented as the mean \pm S.E.M. The maximum time delay between the treatment groups and the control was not significant.

observed here were much lower. The differences in VP amplitudes between the MED64 system and traditional methods are a result of the tissue/microelectrode interface model that is used in the planar microelectrode array but not the traditional methods ${ }^{22}$. Specifically, the position of the reference electrode affects the magnitudes of the VP amplitudes that are recorded ${ }^{23}$. The recording electrodes and reference electrodes of the MED64 probe are arranged in the same planar glass plate ${ }^{24}$, which records the voltage that is generated between the leaf contacting the electrodes and the bath solution $(10 \mathrm{mM} \mathrm{KCl})$ or the standard buffer solution. These results show lower amplitudes than those that were recorded using traditional methods. Although many factors and parameters may affect the performance of the system, the most important factor for the accuracy of MEA is to ensure that the system has an acceptable signal/ noise ratio $^{24,25}$. Although the VP amplitudes were only between $50 \mu \mathrm{V}$ and $400 \mu \mathrm{V}$, we were still able to distinguish the details of the VPs because the noise level of the MED64 system was extremely low $( \pm 17 \mu \mathrm{V})$. We found that there was no effect on the VPs of the leaves and VP propagations using the MED probe with $10 \mathrm{mM} \mathrm{KCl}$ $(\mathrm{n}=38)$ or a standard bath solution $(\mathrm{n}=7)$. Thus, the results are reliable and comparable following non-invasive and nondestructive in situ recording processes using different solutions. Incidentally, Furch et $\mathrm{al}^{26}$ noted that a high concentrations of external $\mathrm{KCl}$ (100 $\mathrm{mM}$ ) evoked a rapid AP-like response; thus, it is important to prevent any breakage from the measured leaf from occurring.

In general, the major long transmission routes of the VPs can be determined using traditional electrophysiology methods, but because of limitations in the numbers and sizes of electrodes, there are few studies examining VP transmission routes at the cellular level, and visualising the transmission of electrical signals using traditional methods is not possible. In the present study, we found that the use of MEA may solve these problems. Our results (Figs. 2d-e and Figs. 3d-e) revealed that the transmission routes of VPs in $H$. annuus leaves did not follow a line or travel from point to point but spread across the surface in multiple directions. The data that are shown in Fig. 2e and Fig. 3e suggest the presence of two types of transmission routes (the blue and red lines) with obvious differences in transmission velocities. Signals travel slowly along the main vein (parallel to the leaf) and much faster along the smaller veins that branch off towards the edges of the leaf. However, signals do not seem to travel directly between the side branches; they arrive at each part of the leaf via the main vein and then follow the branches outward. Considering that different tissues (e.g., veins, epidermis, guard cells, and mesophyll) were covered by the electrode array, it is likely that the VPs spread at different velocities depending on the leaf tissue. Our findings also support the idea that VPs are primarily generated by wounding, which induces a hydraulic wave that is transmitted through the xylem, causing a local electrical response in neighbouring symplastic cells that propagates faster than the response that occurs in the main vein. The signal can be transmitted over short distances from cell to cell through the plasmodesmata, and after it reaches the phloem, it can also propagate over long distances along the sieve-tube plasma membrane $e^{8,27,28}$.

The possibilities and advantages of visualising VP transmission in plants can also be seen in Fig. $2 \mathrm{~d}$ and Fig. $3 \mathrm{~d}$. VP propagation from one side of the detectable region to the other was indicated by the analysed data from the MEA. Complex neuronal networks found in animals do not exist in plants, and many signal patterns, such as bursting or synchronisation that have been widely studied in animal nerves $^{19,29}$ and brains ${ }^{30}$, have not been discovered or investigated in plants in situ. Considering the variations in the features of electrical signals in plants, different information (perhaps corresponding to different environmental stimuli, such as cold, heat, or cutting) might be transmitted through different VP signal types. Woundinginduced VPs have been associated with many responses in plants, including gene expression in tomato ${ }^{31,32}$ and Arabidopsis thaliana ${ }^{33}$, the cessation of mass flow and callose deposition in Cucurbita max$i m a^{34}$. We also observed callose deposition in the present study (see Supplementary Fig. S5). In addition to VPs, the electrical responses to cooling stimulation were also measured using MEA (see Supplementary Fig. S6). Because a MEA can detect electrical signals at multiple sites simultaneously, this technique holds great potential for identifying different signal transformations. Furthermore, when combined with fluorescent dyes or other techniques, it may be applied to study the relationship between electrical activity and physiological responses at multiple sites in the plant.

In many higher plants, VPs have been recorded in leaves ${ }^{21}$, stems ${ }^{35}$, sieve tubes ${ }^{36}$, and petioles ${ }^{37}$ using surface electrodes, aphid stylets, and microelectrodes. Based on these investigations, the major notions regarding VP transmission are focused on the hydraulic 
wave $^{21}$ and chemical-inducing ${ }^{38,39}$ theories, in which the xylem and phloem are suggested to be the long transmission routes for VPs. In the present study, we used conventional inhibitors to investigate the factors influencing VPs and VP propagation. Our results agree with those that have been reported in the literature ${ }^{40}$. In particular, our observations are consistent with reports describing effects of $\mathrm{Ca}^{2+}$ channel blockers ${ }^{26}$ on electrical signals. It has been proposed that an electrical potential wave (EPW)-associated $\mathrm{Ca}^{2+}$ influx may trigger the generation and amplification of countless long-distance signals ${ }^{41,42}$, and these results have encouraged exploration of the ion mechanisms that are involved in electrical activity in the phloem. Moreover, the mapping of VP propagation with high spatiotemporal resolution enables us to determine the distribution features of the VPs and VP propagation patterns. Given the strong research interest in $\mathrm{VPs}^{35,36,42-45}$, this method is a potentially useful tool for the elucidation of the electrical signals that occur following wounding.

In this study, VPs in $H$. annuus leaves were recorded at multiple sites simultaneously using a multi-electrode array. Our results revealed that VPs have a complex surface-transmission pattern in $H$. annuus leaves and indicated the potential of using MEA to visualise VP transmission in plants. Our research suggests that MEA can provide high spatio-temporal resolution recordings to determine the bioelectrical responses of higher plants under stress.

\section{Methods}

Plant materials. The $H$. annuus plants that were used in this study were grown in a greenhouse under a $16 \mathrm{~h}$ light $/ 8 \mathrm{~h}$ dark photoperiod regime at temperatures of $30^{\circ} \mathrm{C}$ and $25^{\circ} \mathrm{C}$ during the day and night, respectively. The growth medium was comprised of $70 \%$ vermiculite and $30 \%$ humus and contained all of the essential nutrients. The plants that were used for the experiments were 2-3 weeks old.

MEA system. We used an MED64 (Alpha MED Scientific Inc., Ibaraki Osaka, Japan) MEA system. This system has 64 planar microelectrodes that are arranged in an $8 \times 8$ array in the centre with four reference electrodes that are symmetrically situated at the four corners of the probe plate (MED-P545A, Alpha MED Scientific Inc.). Each microelectrode size is $50 \mu \mathrm{m} \times 50 \mu \mathrm{m}$, and the distance between adjacent electrodes is $450 \mu \mathrm{m}$. Thus, the array can cover an area of approximately $3.55 \mathrm{~mm} \times 3.55 \mathrm{~mm}$. According to the MED64 Hand Book form Alpha MED Scientific Inc. (Japan), the four reference electrode lines flow together and then converge on a common ground in the MED64 amplifier. Additionally, the user manual suggests that the platinum wire ring functions as an additional reference electrode to suppress noise and stimulus artifacts. A glass ring (I.D. $=22 \mathrm{~mm}$, O.D. $=25 \mathrm{~mm}$, height $=10 \mathrm{~mm}$ ) was attached at the centre of the plate to form a recording chamber. An additional platinum ring $(\mathrm{O} . \mathrm{D} .=21 \mathrm{~mm})$ reference electrode was placed at the bottom of the recording chamber to connect with the ground, but it did not contact the leaves during recording. The sampling frequency and bandwidth were set to $20 \mathrm{kHz}$ and $0.1 \mathrm{~Hz}$ to $100 \mathrm{kHz}$, respectively. The input impedance of the amplifier was $100 \mathrm{M} \Omega$.

Preparation of multi-electrode array. Procedures for the preparation of the introduced multi-electrode probe (MED-P545A, Alpha MED Scientific Inc.) were previously described by Zhao et $\mathrm{al}^{46}$. The MED64 probe was treated with $0.1 \%$ polyethyleneimine (Sigma) in $25 \mathrm{mM}$ borate buffer ( $\mathrm{pH} 8.4$ ) overnight at room temperature ${ }^{46}$. Immediately before use, the probe surface was rinsed three times with sterile distilled water

Coupling of $H$. annuus leaves and MED64 planar microelectrodes. For recording, a leaf was fixed in situ in a recording chamber and placed directly on the multielectrode array, which was connected to the 64-channel system using the MED connector. The chamber was filled with $1 \mathrm{~mL}$ of a $10 \mathrm{mM} \mathrm{KCl}$ solution or standard buffer solution, which immersed the plate electrodes and platinum ring. The recorded part of the leaf was also immersed in the solution. To achieve good contact between the electrodes and the leaf, a U-shaped platinum plate with a grid of nylon threads was placed on the top of the leaf, and a 25 -g weight that was sealed with a plastic membrane was placed on the platinum plate.

Inhibitor Treatment. Inhibitors were added separately to the experimental chamber containing a standard buffer solution of $2 \mathrm{mM} \mathrm{KCl}, 1 \mathrm{mM} \mathrm{CaCl}_{2}, 1 \mathrm{mM} \mathrm{MgCl}_{2}$, $50 \mathrm{mM}$ mannitol, and $2.5 \mathrm{mM}$ MES/ $\mathrm{NaOH}, \mathrm{pH}$ 5.7. The concentrations of inhibitors were as follows: $\mathrm{Ca}^{2+}$ channel inhibitor, $1 \mathrm{mM} \mathrm{LaCl}{ }_{3} ; \mathrm{K}^{+}$channel inhibitor, $10 \mathrm{mM}$ tetraethylammonium chloride; and $\mathrm{H}^{+}$-ATPase inhibitor, $1 \mathrm{mM}$ sodium orthovanadate. Prior to heat stimulation, the duration of incubation for the leaf in the standard solution containing the inhibitors was at least $1 \mathrm{~h}$. After the treatment, all of the recording conditions that were used were the same as those for the untreated control plants.
Stimulation. Thermal stimulation was applied to a nearby leaf, which was separate from the recording leaf. Stimulation was performed by passing a flaming match under the leaf tip for $3 \mathrm{~s}$ in an area measuring approximately $1.5 \mathrm{~cm}^{2}$. The distance between the stimulation point and recording region was $8-10 \mathrm{~cm}$.

Data analysis. Data were collected using MED64 Mobius acquisition software (Witwerx Inc., Tustun, CA, USA) and analysed and visualised using the Matlab software (Matlab R2009). Data are presented as the mean \pm S.E.M. A Student's t-test was used to analyse the differences among groups, and $p<0.05$ was considered significant.

1. Dziubinska, H., Trebacz, K. \& Zawadzki, T. The effect of excitation on the rate of respiration in the liverwort Conocephalum conicum. Physiol. Plantarum 75, 417-423 (1989).

2. Fromm, J. \& Lautner, S. Electrical signals and their physiological significance in plants. Plant Cell Environ. 30, 249-257 (2007).

3. Sibaoka, T. Rapid plant movements triggered by action potentials. Bot. Mag. 104, 73-95 (1991)

4. Maffei, M. \& Bossi, S. Electrophysiology and plant responses to biotic stress. in Plant Electrophysiology. (ed Volkov, A. G.) (Springer Berlin Heidelberg, 2006).

5. Volkov, A. G. et al. Electrotonic and action potentials in the Venus flytrap. J. Plant Physiol. 170, 838-846 (2013).

6. Dziubinska, H., Trebacz, K. \& Zawadzki, T. Transmission route for action potentials and variation potentials in Helianthus annuus L. J. Plant Physiol. 158, 1167-1172 (2001).

7. Oyarce, P. \& Gurovich, L. Evidence for the transmission of information through electric potentials in injured avocado trees. J. Plant Physiol. 168, 103-108 (2011).

8. Fromm, J. \& Lautner, S. Generation, transmission, and physiological effects of electrical signals. in Plant Electrophysiology. (ed Volkov, A. G.) (Springer Berlin Heidelberg, 2012).

9. Yan, X. et al. Research progress on electrical signals in higher plants. Prog. Nat. Sci. 19, 531-541(2009).

10. Davies, E. \& Stanković, B. Electrical signals, the cytoskeleton, and gene expression: A hypothesis on the coherence of the cellular responses to environmental insult. in Communication in Plants. (eds Baluška, F., Mancuso, S. \& Volkmann, D.) (Springer Berlin Heidelberg, 2006).

11. Dziubinska, H., Filek, M., Koscielniak, J. \& Trebacz, K. Variation and action potentials evoked by thermal stimuli accompany enhancement of ethylene emission in distant non-stimulated leaves of Vicia faba minorseedlings. J. Plant Physiol. 160, 1203-1210 (2003).

12. Wildon, D. C. et al. Electrical signalling and systemic proteinase inhibitor induction in the wounded plant. Nature 360, 62-65 (1992).

13. Rhodes, J. D., Thain, J. F. \& Wildon, D. C. Signals and signalling pathways in plant wound responses. in Communication in Plants. (eds Baluška, F., Mancuso, S. \& Volkmann, D) (Springer Berlin Heidelberg, 2006).

14. Normann, R. A., Warren, D. J., Ammermuller, J., Fernandez, E. \& Guillory, S. High-resolution spatio-temporal mapping of visual pathways using multielectrode arrays. Vision Res. 41, 1261-1275 (2001).

15. Thomas, C. A., Springer, P. A., Loeb, G. E., Berwald-Netter, Y. \& Okun, L. M. A miniature microelectrode array to monitor the bioelectric activity of cultured cells. Exp. Cell Res. 74, 61-66 (1972).

16. Spira, M. E. \& Hai, A. Multi-electrode array technologies for neuroscience and cardiology. Nature Nanotech. 8, 83-94 (2013).

17. Charkhkar, H., Knaack, G. L., Gnade, B. E., Keefer, E. W. \& Pancrazio, J. J. Development and demonstration of a disposable low-cost microelectrode array for cultured neuronal network recording. Sensor. Actuat. B-Chem. 161, 655-660 (2012).

18. Kibler, A. B., Jamieson, B. G. \& Durand, D. M. A high aspect ratio microelectrode array for mapping neural activity in vitro. J. Neurosci. Meth. 204, 296-305 (2012).

19. Chu, J. U. et al. Spontaneous synchronized burst firing of subthalamic nucleus neurons in rat brain slices measured on multi-electrode arrays. Neurosci. Res. 72, 324-340 (2012).

20. Masi, E. et al. Spatiotemporal dynamics of the electrical network activity in the root apex. PNAS. 106, 4048-4053 (2009).

21. Stanković, B., Witters, D. L., Zawadzki, T. \& Davies, E. Action potentials and variation potentials in sunflower: an analysis of their relationships and distinguishing characteristics. Physiol. Plantarum 103, 51-58 (1998).

22. Yu, H., Wang, J., Liu, Q., Deng, B. \& Wei, X. Delayed feedback control of bursting synchronization in small-world neuronal networks. Neurocomputing $\mathbf{9 9}, 178-187$ (2013).

23. Egert, U., Heck, D. \& Aertsen, A. Two-dimensional monitoring of spiking networks in acute brain slices. Exp. Brain Res. 142, 268-274 (2002).

24. Oka, H., Shimono, K., Ogawa, R., Sugihara, H. \& Taketani, M. A new planar multielectrode array for extracellular recording: application to hippocampal acute slice. J. Neurosci. Meth. 93, 61-67 (1999).

25. Masi, E., Azzarello, E. \& Mancuso, S. Multielectrode array: A new approach to plant electrophysiology. in Plant Electrophysiology. (ed Volkov, A. G.) (Springer Berlin Heidelberg, 2012).

26. Furch, A. C. U. et al. Sieve element $\mathrm{Ca}^{2+}$ channels as relay stations between remote stimuli and sieve tube occlusion in Vicia faba. The Plant Cell Online 21, 2118-2132 (2009). 
27. Van Bel, A. J. E., Knoblauch, M., Furch, A. C. U. \& Hafke, J. B. Questionsn on phloem biology. 1. Electropotential waves, $\mathrm{Ca}^{2+}$ fluxes and cellular cascades along the propagation pathway. Plant Sci. 181, 210-218 (2011).

28. Dinant, S. \& Lemoine, R. The phloem pathway: new issues and old debates. $C$. $R$. Biol. 333, 307-319 (2010).

29. Hosokawa, C., Kudoh, S. N., Kiyohara, A. \& Taguchi, T. Resynchronization in neuronal network divided by femtosecond laser processing. NeuroReport 19 , 771-775 (2008).

30. Yamazaki, Y.et al. Analysis of cytoarchitecture and electrical activity of the human cerebral and cerebellar cortical slices with multi-electrode array system. Neurosci. Res. 68 Supplement 1, e440-e441 (2010).

31. Davies, E. New functions for electrical signals in plants. New Phytol. 161, 607-610 (2004).

32. Stanković, B. \& Davies, E. Both action potentials and variation potentials induce proteinase inhibitor gene expression in tomato. Febs Lett. 390, 275-279 (1996).

33. Mousavi, S. A. R., Chauvin, A., Pascaud, F., Kellenberger, S. \& Farmer, E. E. Glutamate receptor-like genes mediate leaf-to-leaf wound signalling. Nature 500, 422-426 (2013).

34. Furch, A. C. U., Zimmermann, M. R., Will, T., Hafke, J. B. \& Van Bel, A. J. E. Remote-controlled stop of phloem mass flow by biphasic occlusion in Cucurbita maxima. J. Exp. Bot. 61, 3697-3708 (2010).

35. Stanković, B., Zawadzki, T. \& Davies, E. Characterization of the variation potential in sunflower. Plant Physiol. 115, 1083-1088 (1997).

36. Fromm, J. \& Eschrich, W. Transport processes in stimulated and non-stimulated leaves of Mimosa pudica. Trees 2, 7-17 (1988).

37. Rhodes, J. D., Thain, J. F. \& Wildon, D. C. The pathway for systemic electrical signal conduction in the wounded tomato plant. Planta 200, 50-57 (1996).

38. Malone, M. Rapid, long-distance signal transmission in higher plants. Adv. Bot. Res. 22, 163-228 (1996).

39. Vodeneev, V.et al. The mechanism of propagation of variation potentials in wheat leaves. J. Plant Physiol. 169, 949-954 (2012).

40. Vodeneev, V. A., Akinchits, E. K., Orlova, L. A. \& Sukhov, V. S. The role of $\mathrm{Ca}^{2+}$, $\mathrm{H}^{+}$, and $\mathrm{Cl}^{-}$ions in generation of variation potential in pumpkin plants. Russ. $J$. Plant Physl. 58, 974-981 (2011).

41. Hafke, J. B. \& van Bel, A. J. E. Cellular basis of electrical potential waves along the phloem and impact of coincident $\mathrm{Ca}^{2+}$ fluxes. in Phloem. Molecular Cell Biology, Systemic Communication, Biotic Interactions. (eds Thompson, G. A., van Bel, A. J. E.) (Wiley-Blackwell, 2013).

42. van Bel, A. J. E. et al. Spread the news: systemic dissemination and local impact of $\mathrm{Ca}^{2+}$ signals along the phloem pathway. J. Exper. Bot. 65, 1761-1787 (2014).

43. Stahlberg, R., Cleland, R. E., van Volkenburgh, E. Slow wave potentials-a propagating electrical signal unique to higher plants. in Communication in Plants-Neuronal Aspects of Plant Life. (eds Baluska, F., Mancuso, S., Volkmann, D.) (Springer Berlin Heidelberg, 2006).
44. Stahlberg, R., Cleland, R. E. \& Van Volkenburgh, E. Decrement and amplification of slow wave potentials during their propagation in Helianthus annuus L. shoots. Planta 220, 550-558 (2005).

45. Mancuso, S. Hydraulic and electrical transmission of wound-induced signals in Vitis vinifera. Aust. J. Plant Physiol. 26, 55-61 (1999).

46. Zhao, X.-Y. et al. Nociception-induced spatial and temporal plasticity of synaptic connection and function in the hippocampal formation of rats: a multi-electrode array recording. Mol. Pain 5, 55 (2009).

\section{Acknowledgments}

This research was supported by the National Natural Science Foundation of China (61072016) and the Specialized Research Fund for the Doctoral Program of Higher Education (20130008110035). We thank Mr. Shirakawa Keiichi from Alpha MED Scientific Inc. for his technical assistance.

\section{Author contributions}

L.H. and Z.Y.W. proposed and supervised the project; D.J.Z., L.H. and Z.Y.W. designed the experiments; D.J.Z. performed the experiments. D.J.Z., L.H., Z.Y.W., J.Q.L. and Y.P.J. analysed the data and contributed to the manuscript. D.J.Z. and L.H. wrote the manuscript, and all of the authors participated in the discussions of the results and in the preparation of the manuscript.

\section{Additional information}

Supplementary information accompanies this paper at http://www.nature.com/ scientificreports

Competing financial interests: The authors declare no competing financial interests.

How to cite this article: Zhao, D.-J., Wang, Z.-Y., Huang, L., Jia, Y.-P. \& Leng, J.Q Spatio-temporal mapping of variation potentials in leaves of Helianthus annuus L. seedlings in situ using multi-electrode array. Sci. Rep. 4, 5435; DOI:10.1038/srep05435 (2014).

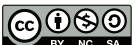

This work is licensed under a Creative Commons Attribution-NonCommercialShareAlike 4.0 International License. The images or other third party material in this article are included in the article's Creative Commons license, unless indicated otherwise in the credit line; if the material is not included under the Creative Commons license, users will need to obtain permission from the license holder in order to reproduce the material. To view a copy of this license, visit http:/ creativecommons.org/licenses/by-nc-sa/4.0/ 